\title{
Efficient formulation for band-structure calculations of two-dimensional phononic-crystal plates
}

\author{
Jin-Chen Hsu and Tsung-Tsong Wu* \\ Institute of Applied Mechanics, National Taiwan University, Taipei 106, Taiwan
}

(Received 2 June 2006; published 6 October 2006)

\begin{abstract}
Based on Mindlin's plate theory and the plane wave expansion method, a formulation is proposed to study the propagation of Lamb waves in two-dimensional phononic-crystal plates. The method is applied to calculate the frequency band structure of a square array of crystalline gold cylinders in an epoxy matrix with a finite thickness. It is found that complete frequency band gaps for Lamb waves between different pass bands are opened up by tuning thickness of the phononic-crystal plate. The influence of plate thickness on the width of complete frequency band gap is calculated and discussed as well; the existence of frequency stop bands is sensitive to the variation of the thickness of the plate. Finally, we note that the proposed method provides a concise and efficient way in analyzing the frequency band structures of phononic-crystal plates in lower bands.
\end{abstract}

DOI: 10.1103/PhysRevB.74.144303 PACS number(s): 63.20. $-\mathrm{e}, 43.20 .+\mathrm{g}, 43.40 .+\mathrm{s}$, 46.40.Cd

\section{INTRODUCTION}

Propagation of acoustic waves in the periodic structures called phononic crystals has received much attention in the last decade. ${ }^{1,2}$ The possibility of achieving a complete frequency band gap, which can forbid the propagation of acoustic waves with any polarization and wave vector, suggests the possible applications of phononic structures as perfect acoustic mirrors, filters, and high efficiency waveguides. Most of the studies concerning phononic crystals focused on investigation of the bulk acoustic waves, ${ }^{3-7}$ and parts of them reported on the acoustic waves localized at the surface of two-dimensional, semi-infinite phononic crystals. ${ }^{8-11}$ Recently, some studies show that another worthwhile subject regarding phononic crystals would be the acoustic wave propagating in a finite thickness plate. Zhang et al. ${ }^{12}$ proposed experimental results of the Lamb wave band gap in the phononic crystals created on thin plates. In their paper, multiple frequency band gaps and narrow pass band within certain frequency band gaps were observed. Sainidou and Stefanou ${ }^{13}$ studied the guided and quasiguided elastic waves in a glass plate coated on one side with a period monolayer of spheres, immersed in water. Researches to study elastic waves in phononic structures with finite thickness, however, remain still little so far. ${ }^{14-16}$ Among the limited existing literature, plane wave expansion (PWE) method is one of the common methods used to analyze this kind of problems. Sigalas and Economou ${ }^{16}$ proposed the classical plate theory based PWE method to calculate the frequency band structures of elastic waves in thin plates with periodically placed inclusions. In their analysis, the thickness/lattice-spacing ratio $(h / a)$ and frequency must be kept in very low ranges to hold the assumptions of classical plate theory. Otherwise, considerable deviations would emerge because the shear deformations of bending of the plate are ignored in the classical plate theory. As the value $h / a$ and frequency increase, the full three-dimensional (3D) PWE method should be considered. The full 3D PWE method, however, requires a huge computation time as a large number of the plane waves were adopted. The dramatic increasing of computation time originates from the procedure for calculating the stress-free boundary-condition determinants in the full 3D PWE model.
This inevitable drawback restricts the choices of material contrast of the constituents of the phononic crystal, or qualitative descriptions can only be offered because a small number of plane waves, such as 49 or less, were considered in most literature.

The purpose of this paper is to calculate and discuss the characteristics of Lamb waves in phononic-crystal plates. To overcome the convergence and large computation time problems in using the full 3D PWE method, we developed an efficient formulation based on Mindlin's plate theory. Our method shows excellent performance for the cases of phononic-crystal plates composed not only of small but also of large material mismatch (mass density and elastic stiffness) of the constituents. Furthermore, the proposed formulation gives explicit physical meaning to identify the polarizations of waves such as flexural, extensional, face-shear, and thickness-shear vibrations in the thin plates.

This paper is organized as follows. In Sec. II, we briefly review the essential of Mindlin's plate theory and derive the PWE method based on Mindlin's theory. In Sec. III, we employ the method developed by us to analyze and discuss the acoustic waves propagating in phononic-crystal plates composed of crystalline gold and epoxy. Finally, some conclusions are given in Sec. IV.

\section{FORMULATION}

In this section, we summarize Mindlin's plate theory ${ }^{17,18}$ and derive an improved plane-wave-expansion formalism by combining with Mindlin's theory to analyze the waves propagating in phononic-crystal plates.

\section{A. Mindlin's plate theory}

Consider a plate with thickness $h$ whose material properties are homogeneous along the thickness direction. We set up the coordinates as follows. Let the $x_{3}$ axis be the thickness direction and directed downward. The $x_{1}-x_{2}$ plane rests in the middle plane of the plate, and the plate surfaces are at $x_{3}$ $= \pm h / 2$. The components of displacement $u_{j},(j=1,2,3)$, are expanded in power series of the thickness coordinate $x_{3}$ 


$$
\begin{aligned}
u_{j}\left(x_{1}, x_{2}, x_{3}, t\right) & =\sum_{n=0}^{\infty} x_{3}^{n} u_{j}^{(n)}\left(x_{1}, x_{2}, t\right) \\
& =u_{j}^{(0)}\left(x_{1}, x_{2}, t\right)+x_{3} u_{j}^{(1)}\left(x_{1}, x_{2}, t\right)+\cdots,
\end{aligned}
$$

where $u_{j}^{(n)}$ is called the $n$th order component of the displacement field. Basically, the zeroth order and the first order terms represent the fundamental modes of the elastic waves in plates (extensional, face-shear, flexural, thickness-shear and thickness-twist modes), and the higher order terms are their overtones. Substitution of the series expression for $u_{j}$ into the variational principle gives the equations of motion of order $n$ by

$$
T_{i j, i}^{(n)}-T_{3 j}^{(n-1)}-\rho \sum_{m=0}^{\infty} H_{n m} \ddot{u}_{j}^{(m)}=0,
$$

where

$$
\begin{gathered}
T_{i j}^{(n)} \equiv \int_{-h / 2}^{h / 2} x_{3}^{n} T_{i j} d x_{3}, \\
H_{n m} \equiv \begin{cases}\frac{h^{m+n+1}}{2^{m+n}(m+n+1)}, & m+n \text { even } \\
0, & m+n \text { odd } .\end{cases}
\end{gathered}
$$

In Eq. (3), $T_{i j}$ are the components of Cauchy stress tensor, and $\rho$ is the mass density. The $n$th order components of strain $S_{i j}^{(n)}$ and stress $T_{i j}^{(n)}$ can be written as

$$
\begin{gathered}
S_{i j}^{(n)}=\frac{1}{2}\left[u_{i, j}^{(n)}+u_{j, i}^{(n)}+(n+1)\left(\delta_{3 j} u_{i}^{(n+1)}+\delta_{3 i} u_{j}^{(n+1)}\right)\right], \\
T_{i j}^{(n)}=c_{i j k l} \sum_{n=0}^{\infty} H_{n m} S_{k l}^{(n)},
\end{gathered}
$$

where $\delta_{i j}$ is Kronecker delta, and $c_{i j k l}$ is the elastic stiffness.

For truncation of the series, only the zeroth and first order components of stress and strain will be retained. By following Cauchy's procedure, ${ }^{19} u_{3}^{(1)}$ is neglected for the free development of the strain $S_{33}^{(0)}\left(=u_{3}^{(1)}\right)$ by setting $T_{33}^{(0)}=0$ in the zeroth order stress. The condition $T_{33}^{(0)}=0$ permits the elimination of $S_{33}^{(0)}$ from the zeroth order stress, with the result

$$
T_{i j}^{(0)}=h g_{i j k l} S_{k l}^{(0)},
$$

where the modified elastic stiffness

$$
g_{i j k l}=c_{i j k l}-c_{i j 33} c_{33 k l} / c_{3333} .
$$

Similarly, all three terms $u_{j}^{(2)}$ are neglected for the free development of the strain $S_{3 j}^{(1)}$ by setting $T_{3 j}^{(1)}=0$. The first order stress, therefore, can be written as

$$
T_{\alpha \beta}^{(1)}=\frac{1}{12} h^{3} \gamma_{\alpha \beta \eta \mu} S_{\eta \mu}^{(1)} ; \quad \alpha, \beta, \eta, \mu=1,2,
$$

where $\gamma_{\alpha \beta \eta \mu}=\frac{\text { cofactor }\left|s_{\alpha \beta \eta \mu}\right|}{\left|s_{\alpha \beta \eta \mu}\right|}, \quad\left|s_{\alpha \beta \eta \mu}\right|=\left|\begin{array}{ccc}s_{1111} & s_{1122} & 2 s_{1112} \\ s_{2211} & s_{2222} & 2 s_{2212} \\ 2 s_{1211} & 2 s_{1222} & 4 s_{1212}\end{array}\right|$,

and $s_{\alpha \beta \eta \mu}$ is the elastic compliance. As the final step in the process of truncation, the strains $S_{13}^{(0)}$ and $S_{23}^{(0)}$ are replaced by $\kappa_{1} S_{13}^{(0)}$ and $\kappa_{3} S_{23}^{(0)}$ in the equations of motion, where $\kappa_{1}$ and $\kappa_{3}$ are correction factors which may be used to adjust the thickness-shear vibrations. In the case of monoclinic symmetry material, the correction factors are given by ${ }^{18}$

$$
\begin{gathered}
\kappa_{1}^{2}=\pi^{2} / 12, \\
\kappa_{3}^{2}=\kappa_{1}^{2}\left[c_{3333}+c_{2323}-\sqrt{\left(c_{3333}-c_{2323}\right)^{2}+4 c_{2323}^{2}}\right] / 2 g_{2323} .
\end{gathered}
$$

Substitution of Eqs. (6) and (8) into Eq. (2), one can obtain the two-dimensional coupled equations of motion of the plate with retaining zeroth and first order terms.

\section{B. PWE method for phononic-crystal plate}

According to Mindlin's plate theory for monoclinic symmetry material summarized in the first part of Sec. II, the equations of motion for plate with retaining zeroth and first order terms can be expanded as follows:

$$
\begin{gathered}
\frac{\partial}{\partial x_{1}}\left[g_{11} u_{1,1}^{(0)}+g_{12} u_{2,2}^{(0)}+\kappa_{3} g_{14}\left(u_{3,2}^{(0)}+u_{2}^{(1)}\right)\right]+\frac{\partial}{\partial x_{2}}\left[\kappa _ { 1 } c _ { 5 6 } \left(u_{3,1}^{(0)}\right.\right. \\
\left.\left.+u_{1}^{(1)}\right)+c_{66}\left(u_{2,1}^{(0)}+u_{1,2}^{(0)}\right)\right]=\rho \ddot{u}_{1}^{(0)} \\
\frac{\partial}{\partial x_{1}}\left[\kappa_{1} c_{56}\left(u_{3,1}^{(0)}+u_{1}^{(1)}\right)+c_{66}\left(u_{2,1}^{(0)}+u_{1,2}^{(0)}\right)\right]+\frac{\partial}{\partial x_{2}}\left[g_{12} u_{1,1}^{(0)}\right. \\
\left.+g_{22} u_{2,2}^{(0)}+\kappa_{3} g_{24}\left(u_{3,2}^{(0)}+u_{2}^{(1)}\right)\right]=\rho \ddot{u}_{2}^{(0)}
\end{gathered}
$$

$$
\begin{aligned}
& \frac{\partial}{\partial x_{1}}\left[\kappa_{1}^{2} c_{55}\left(u_{3,1}^{(0)}+u_{1}^{(1)}\right)+\kappa_{1} c_{56}\left(u_{2,1}^{(0)}+u_{1,2}^{(0)}\right)\right]+\frac{\partial}{\partial x_{2}}\left[\kappa_{3} g_{14} u_{1,1}^{(0)}\right. \\
& \left.\quad+\kappa_{3} g_{24} u_{2,2}^{(0)}+\kappa_{3}^{2} g_{44}\left(u_{3,2}^{(0)}+u_{2}^{(1)}\right)\right]=\rho \ddot{u}_{3}^{(0)} \\
& \frac{h^{3}}{12} \frac{\partial}{\partial x_{1}}\left[\gamma_{11} u_{1,1}^{(1)}+\gamma_{12} u_{2,2}^{(1)}\right]+\frac{h^{3}}{12} \frac{\partial}{\partial x_{2}}\left[\gamma_{66}\left(u_{2,1}^{(1)}+u_{1,2}^{(1)}\right)\right] \\
& \quad-h\left[\kappa_{1}^{2} c_{55}\left(u_{3,1}^{(0)}+u_{1}^{(1)}\right)+\kappa_{1} c_{66}\left(u_{2,1}^{(0)}+u_{1,2}^{(0)}\right)\right]=\frac{\rho h^{3}}{12} \rho \ddot{u}_{1}^{(1)}
\end{aligned}
$$

$$
\begin{aligned}
& \frac{h^{3}}{12} \frac{\partial}{\partial x_{1}}\left[\gamma_{66}\left(u_{2,1}^{(1)}+u_{1,2}^{(1)}\right)\right]+\frac{h^{3}}{12} \frac{\partial}{\partial x_{2}}\left[\gamma_{12} u_{1,1}^{(1)}+\gamma_{22} u_{2,2}^{(1)}\right] \\
& -h\left[\kappa_{3} g_{14} u_{1,1}^{(0)}+\kappa_{3} g_{24} u_{2,2}^{(0)}+\kappa_{3}^{2} g_{44}\left(u_{3,2}^{(0)}+u_{2}^{(1)}\right)\right] \\
& \quad=\frac{\rho h^{3}}{12} \rho \ddot{u}_{2}^{(1)} .
\end{aligned}
$$

In the above expressions, Voigt's notation has been used. Equations (11) are valid for an inhomogeneous plate, in which the material properties vary periodically in the $x_{1}-x_{2}$ 


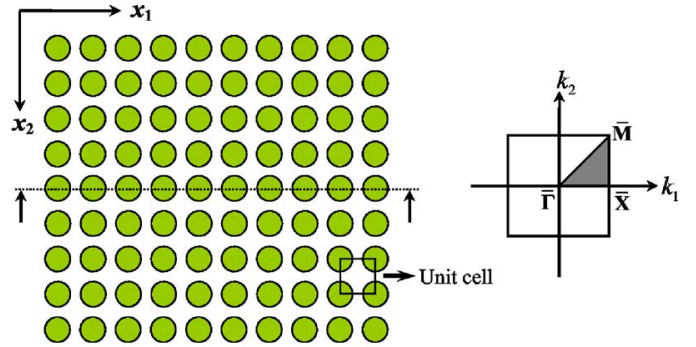

(a)

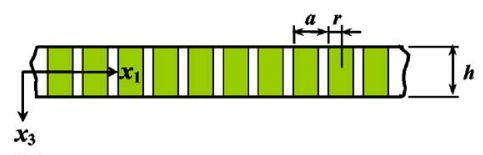

(b)

FIG. 1. (Color online) Top view and cross-section of an infinite two-dimensional phononic-crystal plate. (a) Square lattice and the corresponding first SBZ. (b) Cross section cutting along the dashed lines in (a).

plane. Now, consider an infinite two-dimensional phononiccrystal plate as shown in Fig. 1. The displacement field in a periodic medium must satisfy the Bloch theorem. Therefore, in two-dimensional case, the zeroth and first order components of displacement field can be expressed as

$$
\begin{aligned}
& u_{i}^{(0)}(\mathbf{r}, t)=\sum_{\mathbf{G}} e^{i(\mathbf{k} \cdot \mathbf{x}-\omega t)}\left(e^{i \mathbf{G} \cdot \mathbf{x}} A_{\mathbf{G}}^{i}\right), \quad i=1,2,3, \\
& u_{\alpha}^{(1)}(\mathbf{r}, t)=\sum_{\mathbf{G}} e^{i(\mathbf{k} \cdot \mathbf{x}-\omega t)}\left(e^{i \mathbf{G} \cdot \mathbf{x}} B_{\mathbf{G}}^{\alpha}\right), \quad \alpha=1,2,
\end{aligned}
$$

where $\mathbf{r}=\left(x_{1}, x_{2}, x_{3}\right)=\left(\mathbf{x}, x_{3}\right)$ is the position vector, $\omega$ is the angular frequency, $\mathbf{k}=\left(k_{1}, k_{2}\right)$ is the Bloch wave vector in the surface Brillouin zone (SBZ), and $\mathbf{G}=\left(G_{1}, G_{2}\right)$ is the twodimensional reciprocal lattice vector. $A_{\mathbf{G}}^{i}$ and $B_{\mathbf{G}}^{\alpha}$ are corresponding Fourier coefficients of the zeroth and first order components of displacements, respectively. The periodicity of the structure implies that the material properties $f(\mathbf{x})$ may all be expanded in the Fourier series

$$
f(\mathbf{x})=\sum_{\mathbf{G}} f_{\mathbf{G}} \cdot e^{i \mathbf{G} \cdot \mathbf{x}}
$$

where $f(\mathbf{x})$ is either one of $\rho, g_{p q}, \gamma_{p q}, \kappa_{1} c_{56}, \kappa_{1}^{2} c_{55}, \kappa_{3} g_{14}$, $\kappa_{3} g_{24}$, or $\kappa_{3}^{2} g_{44}$. We note that the Fourier coefficients of those material properties are obtained either by Laurent's rule or by inverse rule according to the procedure of Fourier factorizing a product of two piecewise smooth, bounded, periodic functions. These rules are adopted in this paper for achieving a best convergence. The detailed description about Fourier factorization can be found in Refs. 20 and 21. Substituting Eqs. (12) and (13) into Eq. (11), we obtain a system of equations in the matrix form

$$
\begin{gathered}
\left(\begin{array}{lllll}
M_{\mathbf{G}, \mathbf{G}^{\prime}}^{11} & M_{\mathbf{G}, \mathbf{G}^{\prime}}^{12} & M_{\mathbf{G}, \mathbf{G}^{\prime}}^{13} & M_{\mathbf{G}, \mathbf{G}^{\prime}}^{14} & M_{\mathbf{G}, \mathbf{G}^{\prime}}^{15} \\
M_{\mathbf{G}, \mathbf{G}^{\prime}}^{21} & M_{\mathbf{G}, \mathbf{G}^{\prime}}^{22} & M_{\mathbf{G}, \mathbf{G}^{\prime}}^{23} & M_{\mathbf{G}, \mathbf{G}^{\prime}}^{24} & M_{\mathbf{G}, \mathbf{G}^{\prime}}^{25} \\
M_{\mathbf{G}, \mathbf{G}^{\prime}}^{31} & M_{\mathbf{G}^{\prime}, \mathbf{G}^{\prime}}^{32} & M_{\mathbf{G}, \mathbf{G}^{\prime}}^{33} & M_{\mathbf{G}, \mathbf{G}^{\prime}}^{34} & M_{\mathbf{G}, \mathbf{G}^{\prime}}^{35} \\
M_{\mathbf{G}, \mathbf{G}^{\prime}}^{41} & M_{\mathbf{G}, \mathbf{G}^{\prime}}^{42} & M_{\mathbf{G}, \mathbf{G}^{\prime}}^{43} & M_{\mathbf{G}, \mathbf{G}^{\prime}}^{44} & M_{\mathbf{G}, \mathbf{G}^{\prime}}^{45} \\
M_{\mathbf{G}, \mathbf{G}^{\prime}}^{51} & M_{\mathbf{G}, \mathbf{G}^{\prime}}^{52} & M_{\mathbf{G}, \mathbf{G}^{\prime}}^{53} & M_{\mathbf{G}, \mathbf{G}^{\prime}}^{54} & M_{\mathbf{G}, \mathbf{G}^{\prime}}^{55}
\end{array}\right)\left(\begin{array}{c}
A_{\mathbf{G}^{\prime}}^{1} \\
A_{\mathbf{G}^{\prime}}^{2} \\
A_{\mathbf{G}^{\prime}}^{3} \\
B_{\mathbf{G}^{\prime}}^{1} \\
B_{\mathbf{G}^{\prime}}^{2}
\end{array}\right) \\
\equiv \mathbf{M} \cdot\left(\begin{array}{c}
A_{\mathbf{G}^{\prime}}^{1} \\
A_{\mathbf{G}^{\prime}}^{2} \\
A_{\mathbf{G}^{\prime}}^{3} \\
B_{\mathbf{G}^{\prime}}^{1} \\
B_{\mathbf{G}^{\prime}}^{2}
\end{array}\right)=0 \\
\end{gathered}
$$

While the summation of Eqs. (12) and (13) are truncated up to $n$ in practice, Eq. (14) is reduced to a $5 n \times 5 n$ matrix. Each submatrix $M_{\mathbf{G}, \mathbf{G}^{\prime}}^{l m},(l, m=1-5)$, which are functions of eigenfrequency $\omega$, Bloch wave vector $\mathbf{k}$, reciprocal lattice vector $\mathbf{G}$, and Fourier coefficients $f_{\mathbf{G}}$, is a $n \times n$ matrix. The explicit expressions of the matrix components are listed and explained in the Appendix. As a result, the eigenfrequency of the phononic plate mode can be solved by setting

$$
\operatorname{det}(\mathbf{M})=0 \text {. }
$$

Once the eigenfrequency $\omega_{\mathbf{k}}^{n}$ is obtained from Eq. (15) for specific Bloch vector $\mathbf{k}$ in $n$th band, the relative amplitude of displacements of the eigenmode can also be solved accordingly by substituting the specific value of $\omega_{\mathbf{k}}^{n}$ into Eq. (14).

\section{NUMERICAL RESULTS}

In this section, we use the proposed method to calculate the frequency band structures of the phononic-crystal plates illustrated in Fig. 1. In the system, the crystalline gold (Au) belonging to cubic system serves as the filling material, and epoxy serves as the host material. For comparison purpose, the frequency band structure of the Au/Epoxy plate with thickness $h=0.25 a$ are first calculated using the full 3D PWE method $^{14,22}$ and Mindlin's theory based PWE method, respectively. The crystalline $\mathrm{Au}$ cylinders are arranged as square lattice embedded in the Epoxy host as depicted in Fig. 1. The lattice spacing and the radius of the cylinders are denoted by $a$ and $r$, respectively, and the filling fraction is $F=\pi r^{2} / a^{2}=0.283$. The material constants used in the calculations are $\rho=19300 \mathrm{~kg} / \mathrm{m}^{3}, \quad c_{11}=19.25 \times 10^{10} \mathrm{~N} / \mathrm{m}^{2}, \quad c_{12}$ $=16.3 \times 10^{10} \mathrm{~N} / \mathrm{m}^{2}$, and $c_{44}=4.24 \times 10^{10} \mathrm{~N} / \mathrm{m}^{2}$ for crystalline gold ${ }^{23}$ and $\rho=1180 \mathrm{~kg} / \mathrm{m}^{3}, c_{11}=7.58 \times 10^{9} \mathrm{~N} / \mathrm{m}^{2}$, and $c_{12}=4.42 \times 10^{9} \mathrm{~N} / \mathrm{m}^{2}$, for epoxy. Figure 2 displays the frequency band structure of the Au/Epoxy phononic-crystal plate, solid lines represent the results obtained by using the full 3D PWE method, and dots denote the results calculated by using Mindlin's theory based PWE method. Due to the large computation time required for the full 3D PWE method, the number of plane waves is restricted to 81 . We note that the CPU times to calculate the frequency band structure along the boundary of the irreducible part of the 


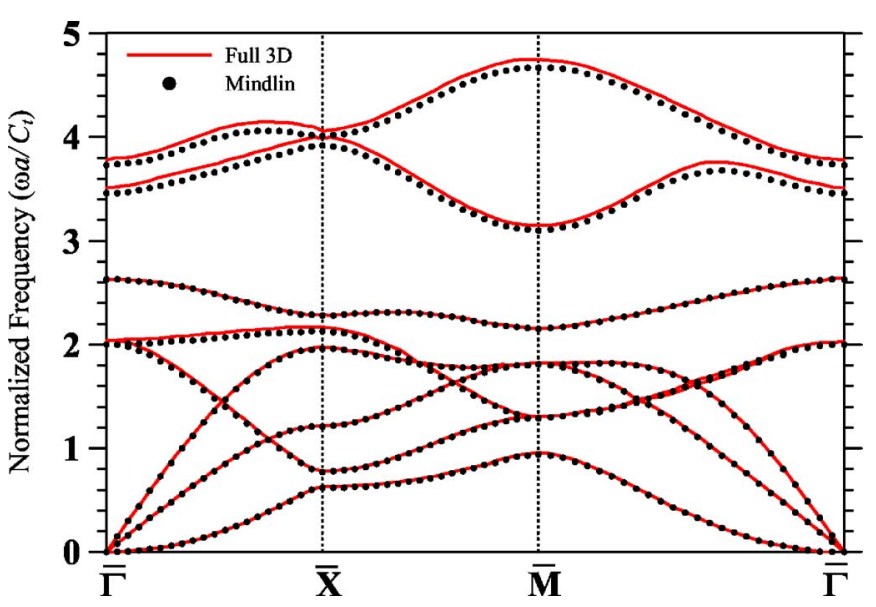

FIG. 2. (Color online) Band structure of the infinite twodimensional phononic-crystal plate with square lattice. The plate consists of crystalline Au cylinders and epoxy. The filling fraction and plate thickness are $F=0.283$ and $h=0.25 a$, respectively. Results are obtained by using the full 3D PWE method (solid lines) and by using Mindlin's theory based PWE method (dots). 81 plane waves are used in the calculations. The quantity $C_{t}$ is transverse wave velocity of epoxy given by $\sqrt{c_{44} / \rho}$.

first SBZ (40500 grid points are included) are about $250 \mathrm{~h}$ for the full 3D PWE method and only $2 \mathrm{~h}$ for Mindlin's theory based PWE method with 81 plane waves on a personal computer equipped with Intel Pentium4 CPU of $2.80 \mathrm{GHz}$ and $512 \mathrm{MB}$ memory. In the figure, we observe that the two methods result in good agreement of numerical results. The maximum difference is about $1.6 \%$ at eighth band in the calculated interval of normalized frequency. Another indispensable observation in Fig. 2 is that there is no missing frequency band in the calculated frequency range for this case. However, Mindlin's theory with retaining zeroth and first order terms in Eq. (1) includes only five plate modes. If higher frequency ranges are considered, those higher plate modes and good accuracy will not be preserved. Therefore, the frequency ranges and the thicknesses of the phononic-crystal plates must be adequately restricted when Mindlin's plate theory based PWE method is used. From the above analysis, the PWE method based on Mindlin's theory exhibits satisfactory accuracy and less computation time. These results suggest that the proposed method can serve as a quick and good predicting tool in the design of a phononiccrystal plate in lower bands.

Moreover, good convergence of the numerical results should be obtained by implementing the calculations with a large number of plane waves when a phononic-crystal plate consisting of materials with large contrast is analyzed. 81 plane waves used in the calculations of Fig. 2 do not provide satisfactory convergence. The full 3D PWE method, however, applied to the phononic-crystal plate problem is not practical for computation time consideration when the number of the plane waves is large. Therefore, with Mindlin's theory based PWE method, 441 plane waves are used to recalculate the dispersion relations of our example, and the result is shown in Fig. 3. In the figure we can observe that a complete frequency band gap exists between the sixth and

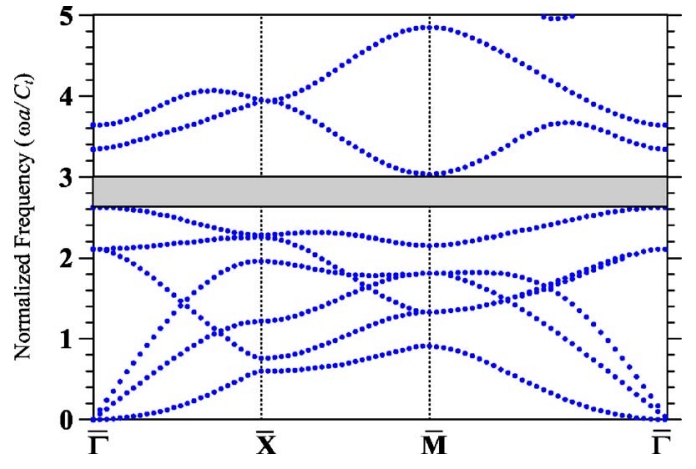

FIG. 3. (Color online) Band structure of the infinite twodimensional phononic-crystal plate calculated with 441 plane waves. The plate is composed of a square array of Au cylinders embedded in epoxy. The filling fraction is 0.283 , and the thickness is $0.25 a$.

seventh frequency bands, and extends in normalized frequency from 2.62 to 3.03 . The ratio of gap width to midgap frequency, therefore, is $\Delta \omega / \omega_{m}=14.6 \%$ for the first complete frequency band gap. We note that the transmission properties for phononic-crystal plate are unobtainable by our means. However, justification of frequency band gaps of phononiccrystal plates from the transmission properties is needed for an approach to reality or experiment. Also, the complete frequency band gaps for the structures of phononic-crystal plates with finite width, similar to a strip structure, demand for further examination with involving the edge conditions. Other methods such as finite-difference time-domain method and multiple-scattering theory ${ }^{24}$ should be suitable to calculate the transmission properties of Lamb waves in both of the plate structures.

Furthermore, in the phononic-crystal plate, the frequency band structure could be quite different from that of an infinite phononic crystal for bulk waves because the waves confined in the finite thickness plate result in an acute dispersion effect in low frequency region by supporting the flexural and thickness-shear vibrations. Therefore, the ratio $h / a$ can be another one of influential parameters on opening the complete band gap and band shifting in the frequency band structure of a phononic-crystal plate. Figure 4 shows the frequency band structure corresponding to the phononic-crystal plate with a smaller thickness $h=0.175 a$; other parameters employed in the calculations are remained unchanged. In Fig. 4 another complete frequency band gap in this thinner phononic-crystal plate is found, and the ratio of gap width to midgap frequency is $\Delta \omega / \omega_{m}=9.3 \%$. Comparing Fig. 4 with Fig. 3, we find that the complete frequency band gap between sixth and seventh frequency bands is closed, and another complete frequency band gap is opened up between fifth and sixth frequency bands, and in the lower frequency range, by tuning down the thickness of the phononic-crystal plate. In others words, for the case of Au/Epoxy phononiccrystal plate, wider and higher complete frequency band gap is obtained in a thicker phononic-crystal plate, and lower complete frequency band gap can be created in a thin phononic-crystal plate.

Figure 5 displays the thickness dependence of the first complete frequency band gap width in the Au/Epoxy 


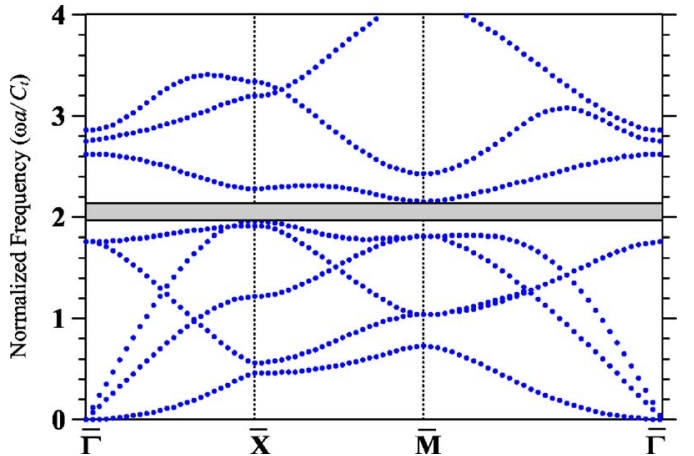

FIG. 4. (Color online) Band structure of the infinite twodimensional phononic-crystal plate calculated with 441 plane waves. The plate is the same as for Fig. 3, but the thickness is changed to $0.175 a$.

phononic-crystal plate. The filling fraction is set at $F$ $=0.283$. In the thin plate region $(h / a \leqslant 0.20)$, we find that complete frequency band gaps exist between the fifth and sixth frequency bands sustains a short thickness/latticespacing range, $\Delta h / a \cong 0.075$, and closes down when the thickness $h \leqslant 0.125 a$ or $h \geqslant 0.20 a$. The local maximum band gap width takes place at $h=0.175 a$. In the thicker plate region $(h / a \geqslant 0.20)$, the complete frequency band gaps appear between the sixth and seventh frequency bands and lie in the relatively higher frequency regions; the frequency band gap width increases progressively with the increase of thickness when $h \geqslant 0.20 a$.

It is understood that the formation of wide frequency band gaps for bulk acoustic waves propagating in the infinite phononic crystals originates from the interaction between the rigid-body resonances of individual fillers and waves propagating in an effective homogeneous medium, and the coalescence with the Bragg gaps..$^{25,26}$ Correspondingly, in a phononic-crystal plate, hybridization of the rigid-body resonance of the individual circular $\mathrm{Au}$ plates with the propaga-

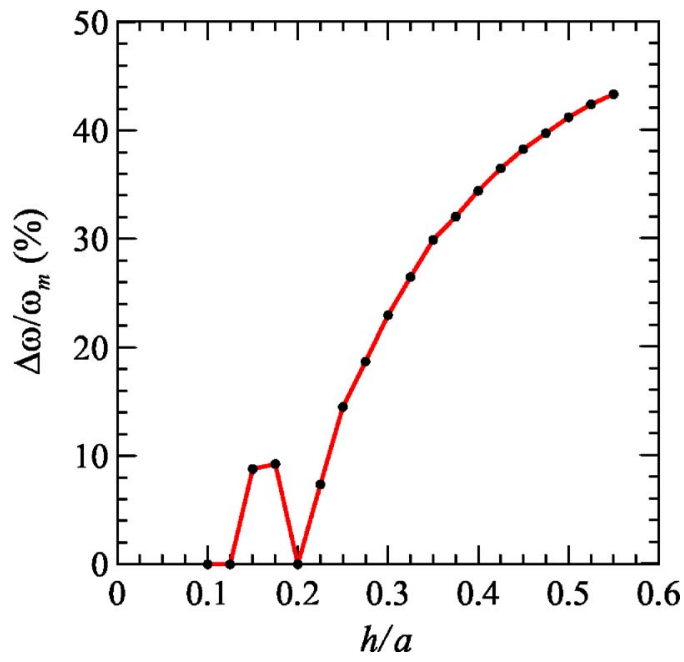

FIG. 5. (Color online) The width of the complete frequency band gap over midgap frequency for the phononic-crystal plate of $\mathrm{Au}$ cylinders in epoxy as a function of the thickness. The filling fraction is set at 0.283 . tion in the effective homogeneous medium corresponding to the periodic plate results in the complete frequency band gap of Lamb waves to exist. Change in the thickness of the plate dramatically changes the scattering properties of the individual circular plates and thus their resonances; therefore, the frequency band structure of the phononic-crystal plate is sensitive to the variation of the thickness. Eventually, the eigenfrequencies of resonance states can be shifted by tuning the thickness of the plate to create complete frequency band gaps between different frequency bands as we have shown in Figs. 3 and 4.

\section{CONCLUSION}

Based on Mindlin's plate theory and the plane wave expansion method, we have studied the propagation behavior for the lower bands of Lamb waves in two-dimensional phononic-crystal plates consisting of square array of crystalline gold cylinders in the epoxy matrix. The secular equations for calculating the frequency band structures and vibration modes have been derived, and the explicit expression for each component in the equation has also been given. The numerical results are compared with those obtained from full 3D PWE method, and complete frequency band gaps for Lamb waves in the Au/Epoxy phononic-crystal plate have been found. Numerical results also show that the complete frequency band gaps between different pass bands can be created by tuning the thickness of the plates. We find that the complete frequency band gap opens up in a small range of thickness for thin plate, and then temporarily closes down until another frequency band gap between higher frequency bands opens up in the thicker plate range. The eigenfrequency of the resonance state depends significantly on the plate thickness; the frequency band gap width and location, therefore, are influenced by the chosen thickness.

\section{ACKNOWLEDGMENT}

The authors gratefully acknowledge the financial support from the National Science Council, Taiwan (Grant No. NSC 94-2212-E-002-040).

\section{APPENDIX}

For convenience, we define five parameters as the products of correction-factor and material property as follows:

$$
\begin{gathered}
K_{156}=\kappa_{1} c_{56}, \quad K_{314}=\kappa_{3} g_{14}, \quad K_{324}=\kappa_{3} g_{24}, \\
R_{155}=\kappa_{1}^{2} c_{55}, \quad R_{344}=\kappa_{3}^{2} g_{44} .
\end{gathered}
$$

The expressions of elements of the submatrices $M_{\mathbf{G}, \mathbf{G}^{\prime}}^{l m}$ in matrix $\mathbf{M}$ are

$$
\begin{gathered}
M_{\mathbf{G}, \mathbf{G}^{\prime}}^{11}=\omega^{2} \rho_{\mathbf{G}-\mathbf{G}^{\prime}}-\left(G_{1}+k_{1}\right)\left(G_{1}^{\prime}+k_{1}\right) g_{\mathbf{G}-\mathbf{G}^{\prime}}^{11} \\
-\left(G_{2}+k_{2}\right)\left(G_{2}^{\prime}+k_{2}\right) c_{\mathbf{G}-\mathbf{G}^{\prime}}^{66}, \\
M_{\mathbf{G}, \mathbf{G}^{\prime}}^{12}=-\left(G_{1}+k_{1}\right)\left(G_{2}^{\prime}+k_{2}\right) g_{\mathbf{G}-\mathbf{G}^{\prime}}^{12} \\
-\left(G_{2}+k_{2}\right)\left(G_{1}^{\prime}+k_{1}\right) c_{\mathbf{G}-\mathbf{G}^{\prime}}^{66},
\end{gathered}
$$




$$
\begin{aligned}
& M_{\mathbf{G}, \mathbf{G}^{\prime}}^{13}=-\left(G_{1}+k_{1}\right)\left(G_{2}^{\prime}+k_{2}\right) K_{\mathbf{G}-\mathbf{G}^{\prime}}^{314} \\
& M_{\mathbf{G}, \mathbf{G}^{\prime}}^{35}=i\left(G_{2}+k_{2}\right) R_{\mathbf{G}-\mathbf{G}^{\prime}}^{344}, \\
& -\left(G_{2}+k_{2}\right)\left(G_{1}^{\prime}+k_{1}\right) K_{\mathbf{G}-\mathbf{G}^{\prime}}^{156}, \\
& M_{\mathbf{G}, \mathbf{G}^{\prime}}^{41}=-i h\left(G_{2}^{\prime}+k_{2}\right) K_{\mathbf{G}-\mathbf{G}^{\prime}}^{156}, \\
& M_{\mathbf{G}, \mathbf{G}^{\prime}}^{14}=i\left(G_{2}+k_{2}\right) K_{\mathbf{G}-\mathbf{G}^{\prime}}^{156} \\
& M_{\mathbf{G}, \mathbf{G}^{\prime}}^{15}=i\left(G_{1}+k_{1}\right) K_{\mathbf{G}-\mathbf{G}^{\prime}}^{314} \\
& M_{\mathbf{G}, \mathbf{G}^{\prime}}^{21}=-\left(G_{1}+k_{1}\right)\left(G_{2}^{\prime}+k_{2}\right) c_{\mathbf{G}-\mathbf{G}^{\prime}}^{66} \\
& -\left(G_{2}+k_{2}\right)\left(G_{1}^{\prime}+k_{1}\right) g_{\mathbf{G}-\mathbf{G}^{\prime}}^{12}, \\
& M_{\mathbf{G}, \mathbf{G}^{\prime}}^{22}=\omega^{2} \rho_{\mathbf{G}-\mathbf{G}^{\prime}}-\left(G_{1}+k_{1}\right)\left(G_{1}^{\prime}+k_{1}\right) c_{\mathbf{G}-\mathbf{G}^{\prime}}^{66} \\
& -\left(G_{2}+k_{2}\right)\left(G_{2}^{\prime}+k_{2}\right) g_{\mathbf{G}-\mathbf{G}^{\prime}}^{22}, \\
& M_{\mathbf{G}, \mathbf{G}^{\prime}}^{23}=-\left(G_{1}+k_{1}\right)\left(G_{1}^{\prime}+k_{1}\right) K_{\mathbf{G}-\mathbf{G}^{\prime}}^{155} \\
& -\left(G_{2}+k_{2}\right)\left(G_{2}^{\prime}+k_{2}\right) K_{\mathbf{G}-\mathbf{G}^{\prime}}^{324}, \\
& M_{\mathbf{G}, \mathbf{G}^{\prime}}^{24}=i\left(G_{1}+k_{1}\right) K_{\mathbf{G}-\mathbf{G}^{\prime}}^{156} \\
& M_{\mathbf{G}, \mathbf{G}^{\prime}}^{25}=i\left(G_{2}+k_{2}\right) K_{\mathbf{G}-\mathbf{G}^{\prime}}^{324} \\
& M_{\mathbf{G}, \mathbf{G}^{\prime}}^{31}=-\left(G_{1}+k_{1}\right)\left(G_{2}^{\prime}+k_{2}\right) K_{\mathbf{G}-\mathbf{G}^{\prime}}^{155} \\
& -\left(G_{2}+k_{2}\right)\left(G_{1}^{\prime}+k_{1}\right) K_{\mathbf{G}-\mathbf{G}^{\prime}}^{314}, \\
& M_{\mathbf{G}, \mathbf{G}^{\prime}}^{32}=-\left(G_{1}+k_{1}\right)\left(G_{1}^{\prime}+k_{1}\right) K_{\mathbf{G}-\mathbf{G}^{\prime}}^{156} \\
& -\left(G_{2}+k_{2}\right)\left(G_{2}^{\prime}+k_{2}\right) K_{\mathbf{G}-\mathbf{G}^{\prime}}^{324}, \\
& M_{\mathbf{G}, \mathbf{G}^{\prime}}^{42}=-i h\left(G_{1}^{\prime}+k_{1}\right) K_{\mathbf{G}-\mathbf{G}^{\prime}}^{156} \\
& M_{\mathbf{G}, \mathbf{G}^{\prime}}^{43}=-i h\left(G_{1}^{\prime}+k_{1}\right) K_{\mathbf{G}-\mathbf{G}^{\prime}}^{155}, \\
& M_{\mathbf{G}, \mathbf{G}^{\prime}}^{44}=\frac{h^{3} \omega^{2}}{12} \rho_{\mathbf{G}-\mathbf{G}^{\prime}}-\frac{h^{3}}{12}\left(G_{1}+k_{1}\right)\left(G_{1}^{\prime}+k_{1}\right) \gamma_{\mathbf{G}-\mathbf{G}^{\prime}}^{11} \\
& -\frac{h^{3}}{12}\left(G_{2}+k_{2}\right)\left(G_{2}^{\prime}+k_{2}\right) \gamma_{\mathbf{G}-\mathbf{G}^{\prime}}^{66}-h R_{\mathbf{G}-\mathbf{G}^{\prime}}^{155} \\
& M_{\mathbf{G}, \mathbf{G}^{\prime}}^{45}=-\frac{h^{3}}{12}\left(G_{1}+k_{1}\right)\left(G_{2}^{\prime}+k_{2}\right) \gamma_{\mathbf{G}-\mathbf{G}^{\prime}}^{12} \\
& -\frac{h^{3}}{12}\left(G_{2}+k_{2}\right)\left(G_{1}^{\prime}+k_{1}\right) \gamma_{\mathbf{G}-\mathbf{G}^{\prime}}^{66}, \\
& M_{\mathbf{G}, \mathbf{G}^{\prime}}^{51}=-i h\left(G_{1}^{\prime}+k_{1}\right) K_{\mathbf{G}-\mathbf{G}^{\prime}}^{314} \\
& M_{\mathbf{G}, \mathbf{G}^{\prime}}^{52}=-i h\left(G_{2}^{\prime}+k_{2}\right) K_{\mathbf{G}-\mathbf{G}^{\prime}}^{324}, \\
& M_{\mathbf{G}, \mathbf{G}^{\prime}}^{53}=-i h\left(G_{2}^{\prime}+k_{2}\right) R_{\mathbf{G}-\mathbf{G}^{\prime}}^{344}, \\
& M_{\mathbf{G}, \mathbf{G}^{\prime}}^{54}=-\frac{h^{3}}{12}\left(G_{1}+k_{1}\right)\left(G_{2}^{\prime}+k_{2}\right) \gamma_{\mathbf{G}-\mathbf{G}^{\prime}}^{66} \\
& -\frac{h^{3}}{12}\left(G_{2}+k_{2}\right)\left(G_{1}^{\prime}+k_{1}\right) \gamma_{\mathbf{G}-\mathbf{G}^{\prime}}^{12}, \\
& M_{\mathbf{G}, \mathbf{G}^{\prime}}^{33}=\omega^{2} \rho_{\mathbf{G}-\mathbf{G}^{\prime}}-\left(G_{1}+k_{1}\right)\left(G_{1}^{\prime}+k_{1}\right) R_{\mathbf{G}-\mathbf{G}^{\prime}}^{155} \\
& -\left(G_{2}+k_{2}\right)\left(G_{2}^{\prime}+k_{2}\right) R_{\mathbf{G}-\mathbf{G}^{\prime}}^{344}, \\
& M_{\mathbf{G}, \mathbf{G}^{\prime}}^{34}=i\left(G_{1}+k_{1}\right) R_{\mathbf{G}-\mathbf{G}^{\prime}}^{155}, \\
& M_{\mathbf{G}, \mathbf{G}^{\prime}}^{55}=\frac{h^{3} \omega^{2}}{12} \rho_{\mathbf{G}-\mathbf{G}^{\prime}}-\frac{h^{3}}{12}\left(G_{1}+k_{1}\right)\left(G_{1}^{\prime}+k_{1}\right) \gamma_{\mathbf{G}-\mathbf{G}^{\prime}}^{66} \\
& -\frac{h^{3}}{12}\left(G_{2}+k_{2}\right)\left(G_{2}^{\prime}+k_{2}\right) \gamma_{\mathbf{G}-\mathbf{G}^{\prime}}^{22}-h R_{\mathbf{G}-\mathbf{G}^{\prime}}^{344}
\end{aligned}
$$

*Corresponding author. Email address: wutt@ndt.iam.ntu.edu.tw

${ }^{1}$ M. S. Kushwaha, P. Halevi, L. Dobrzynski, and B. DjafariRouhani, Phys. Rev. Lett. 71, 2022 (1993).

${ }^{2}$ M. M. Sigalas and E. N. Economou, J. Sound Vib. 158, 377 (1992).

${ }^{3}$ J. O. Vasseur, P. A. Deymier, B. Chenni, B. Djafari-Rouhani, L. Dobrzynski, and D. Prevost, Phys. Rev. Lett. 86, 3012 (2001).

${ }^{4}$ S. Yang, J. H. Page, Z. Liu, M. L. Cowan, C. T. Chan, and P. Sheng, Phys. Rev. Lett. 93, 024301 (2001).

${ }^{5}$ A. Khelif, M. Wilm, V. Laude, S. Ballandras, and B. DjafariRouhani, Phys. Rev. E 69, 067601 (2004).

${ }^{6}$ L. Feng, X.-P. Liu, Y.-B. Chen, Z.-P. Huang, Y.-W. Mao, Y.-F. Chen, J. Zi, and Y.-Y. Zhu, Phys. Rev. B 72, 033108 (2005).
${ }^{7}$ I. E. Psarobas, N. Stefanou, and A. Modinos, Phys. Rev. B 62, 278 (2000).

${ }^{8}$ Y. Tanaka and S. I. Tamura, Phys. Rev. B 58, 7958 (1998).

${ }^{9}$ T.-T. Wu, Z.-C. Hsu, and Z.-G. Huang, Phys. Rev. B 71, 064303 (2005).

${ }^{10}$ T.-T. Wu, Z.-G. Huang, and S. Lin, Phys. Rev. B 69, 094301 (2004).

${ }^{11}$ J.-C. Hsu and T.-T. Wu, IEEE Trans. Ultrason. Ferroelectr. Freq. Control 53, 1169 (2006).

${ }^{12}$ X. Zhang, T. Jackson, E. Lafond, P. Deymier, and J. Vasseur, Appl. Phys. Lett. 88, 041911 (2006).

${ }^{13}$ R. Sainidou and N. Stefanou, Phys. Rev. B 73, 184301 (2006).

${ }^{14}$ M. Wilm, S. Ballandras, V. Laude, and T. Pastureaud, J. Acoust. 
Soc. Am. 112, 943 (2002).

${ }^{15}$ J.-J. Chen, K.-W. Zhang, J. Gao, and J.-C. Cheng, Phys. Rev. B 73, 094307 (2006).

${ }^{16}$ M. M. Sigalas and E. N. Economou, J. Appl. Phys. 75, 2845 (1994).

${ }^{17}$ R. D. Mindlin, J. Appl. Phys. 22, 316 (1951).

${ }^{18}$ R. D. Mindlin, Q. Appl. Math. 19, 51 (1961).

${ }^{19}$ A. L. Cauchy, Exercises de mathématiques (Chez De Bure Frères, Paris, 1826-1830), Vol. 4, p. 1; Oeuvres Ser. 2 (1891), Vol. 19, p. 9.

${ }^{20}$ Y. Cao, Z. Hou, and Y. Liu, Phys. Lett. A 327, 247 (2004).
${ }^{21}$ L. Li, J. Opt. Soc. Am. A 13, 1879 (1996).

${ }^{22}$ J.-C. Hsu and T.-T. Wu, J. Mech. (to be published).

${ }^{23}$ D. Royer and E. Dieulesaint, Elastic Waves in Solids I (SpringerVerlag, Berlin, 2000).

${ }^{24}$ I. E. Psarobas, N. Stefanou, and A. Modinos, Phys. Rev. B 62, 5536 (2000).

${ }^{25}$ R. Sainidou, N. Stefanou, and A. Modinos, Phys. Rev. B 66, 212301 (2002).

${ }^{26}$ H. Zhao, Y. Liu, G. Wang, J. Wen, D. Yu, X. Han, and X. Wen, Phys. Rev. B 72, 012301 (2006). 